\title{
An experiment on wind energy
}

\author{
Vincenzo Lombardo ${ }^{1}$, Emilio Fiordilino ${ }^{1}$, \\ Aurelio Agliolo Gallitto ${ }^{1,3}$ and Pasquale Aglieco ${ }^{2}$ \\ ${ }^{1}$ Dipartimento di Fisica, Università di Palermo, Via Archirafi 36, I-90123 Palermo, Italy \\ 2 Istituto Tecnico Industriale Statale 'Alessandro Volta', Passaggio dei Picciotti 1, I-90123 \\ Palermo, Italy \\ E-mail: aurelio.agliologallitto@unipa.it
}

\begin{abstract}
We discuss an experiment on wind energy performed with home-made apparatus. The experiment reproduces a laboratory windmill, which can pump water from a lower level to a higher one. By measuring the gain of the gravitational potential energy of the pumped water, one can determine the power extracted from the wind. The activity was carried out with high-school students, in the framework of the Italian National Plan for Scientific Degrees-Physics. The proposed experiment allows teachers to discuss renewable energy sources with students whose knowledge of physics is limited to mechanics. It gives students the possibility to gain experience with energy and to increase their awareness of this renewable energy source.
\end{abstract}

\section{Introduction}

Wind energy has been exploited since prehistory, with early historical applications including sailing vessels and windmills [1]. Windmills were used to pump water from at least the 9th century and were later used extensively in Europe. Since the 14th century, in the Netherlands windmills were built to pump out the redundant water from the polder, land reclaimed from the sea or rivers and protected by dykes, to improve agricultural development.

Early immigrants to the United States of America brought with them the technology of windmills from Europe; in 1930, an estimated 600000 units were in use, so that a multi-bladed wind turbine atop a bar-lattice tower was a characteristic of the rural landscape [2].

Wind energy can be obtained everywhere; it is renewable and endless. However, it has gained economic significance only recently and

\footnotetext{
3 Author to whom any correspondence should be addressed
}

today one can see everywhere modern wind farms that transform the kinetic energy of wind into electric energy, based on the well known FaradayNeumann-Lenz law. Wind turbines allow one to fulfil any energy need at any level of growth and to help significantly developing countries or isolated communities, for whom the installation of miniturbines is more convenient than extension of the national electric grid [3].

The inclusion of wind energy topics in school programmes, as well as of other renewableenergy-source topics $[4,5]$, could give students the possibility to gain experience with energysupply systems and could raise their awareness of the benefits that can be obtained in daily life by using renewable energy sources.

In this paper, we discuss an experiment on wind energy performed using home-made apparatus and carried out with high-school students, during the activities of the Italian National Plan for Scientific Degrees-Physics. The activities were carried out with the aim 


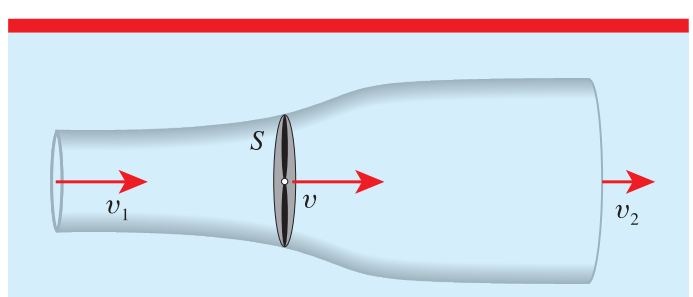

Figure 1. Fluid flow field of the air flux that goes through the rotor; $S$ is the area swept by the rotor blades; $v_{1}>v>v_{2}$.

of increasing the interest of young people in scientific studies [4-7]. Further activity carried out with high-school students, as well as secondary-school students, has also been put forward, such as, for example, the so-called 'School adopts an experiment' programme [8, 9]. Here, we propose a quantitative experiment to exploit the wind's kinetic energy to increase the potential gravitational energy of some amount of water by pumping it from a lower level to a higher one.

\section{Theoretical description}

To exploit wind energy, the kinetic energy of moving air, a wind turbine is necessary. This is a device that converts the kinetic energy from the wind into rotational mechanical energy. If the mechanical energy is used to produce electricity, the device is often called a wind generator. If the mechanical energy is used to drive machinery, such as for grinding grain or pumping water, the device is called a windmill or wind pump [10].

In order to explain the physical origin of the wind energy, let us consider an air flux tube in which a blade rotor is located, as illustrated in figure 1 .

By applying Bernoulli's equation between two sections of the flow tube and considering the air as an ideal fluid, one has

$$
p_{1}+\frac{1}{2} \rho v_{1}^{2}+\rho g h_{1}=p_{2}+\frac{1}{2} \rho v_{2}^{2}+\rho g h_{2},
$$

where $p_{1}, v_{1}$ and $h_{1}$ are respectively the pressure, speed and height of the fluid in the section in front of the rotor; $p_{2}, v_{2}$ and $h_{2}$ are respectively the pressure, speed and height of the fluid in the section behind the rotor; $\rho=m / V$ is the air density and $g$ the gravitational acceleration; the air density at atmospheric pressure, $p_{0}$, and at room temperature, $T_{0} \approx 20{ }^{\circ} \mathrm{C}$, is $\rho \approx 1.2 \mathrm{~kg} \mathrm{~m}^{-3}$ (the density of water is $\rho_{w}=1000 \mathrm{~kg} \mathrm{~m}^{-3}$ ).

In the presence of the rotor, part of the kinetic energy of the air flow is extracted by the rotor. The extracted power $P$ is given by the product of the extracted energy per volume unit times the volume of air that flows through the rotor in the time unit. By equating the kinetic energy of the air lost when it goes through the rotor with the energy gained by the rotor [11], one easily obtains the following relation:

$$
\frac{1}{2} \rho S v\left(v_{1}^{2}-v_{2}^{2}\right)=\rho S v^{2}\left(v_{1}-v_{2}\right),
$$

which gives $v=\left(v_{1}+v_{2}\right) / 2$. From this analysis, it results that the velocity of the air at the plane of the rotor blades is always the averaged value of the velocity of the upstream air and that of the downstream air. Therefore, the extracted power is

$$
P=\frac{P_{\text {nom }}}{2}\left(1+\frac{v_{2}}{v_{1}}\right)^{2}\left(1-\frac{v_{2}}{v_{1}}\right),
$$

where we have defined the nominal theoretical power $P_{\text {nom }}$ that can be extracted from the wind as

$$
P_{\text {nom }}=\frac{1}{2} \rho S v_{1}^{3} .
$$

The power that can be transferred from the wind to the rotor as a function of the ratio $v_{2} / v_{1}$ is plotted in figure 2. The maximum power that can be transferred from the wind to the rotors is obtained for $v_{2}=v_{1} / 3$, therefore the power extracted from the wind is

$$
P_{\text {max }}=\frac{16}{27} \frac{1}{2} \rho S v_{1}^{3}=\frac{16}{27} P_{\text {nom }} \approx 0.59 P_{\text {nom }} .
$$

The result of equation (5) is known as Betz's law, from the German physicist Albert Betz, who first formulated this law [12]. The physical interpretation of Betz's law comes out from the fact that the air flowing through the rotor cannot reduce its velocity to zero, because it must maintain a constant flux (stationary air flow) and because otherwise it would accumulate just after the rotor blades. All of the upstream wind power cannot be extracted by the rotor, as some power is left in the downstream air that continues to move with reduced speed. Consequently, the kinetic energy of the wind cannot be transferred completely to the rotor, but a part of it remains 


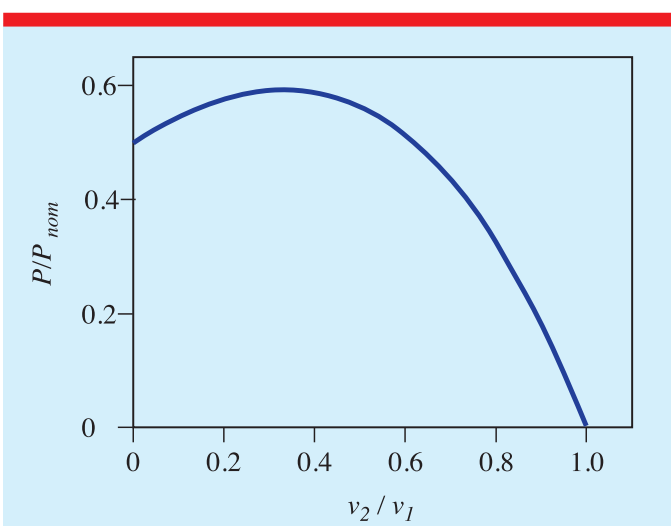

Figure 2. Fraction of extracted power as a function of the ratio $v_{2} / v_{1}$.

for the air to escape from the rotor. This is a theoretical limit that cannot be overcome. It is worth noting that often it is convenient to define the nominal energy flux as $\Phi_{\text {nom }}=\frac{1}{2} \rho v^{3}$ ( $v$ is the wind velocity), which is measured in $\mathrm{W} \mathrm{m}^{-2}$ in the SI unit system.

So far, we have only considered the power that the rotor can ideally extract from the wind. However, during the energy conversion process, due to the mechanical movement of different parts of the apparatus, friction wastes energy. Furthermore, parasitic aerodynamic effects, such as turbulence and swirl, during the air flow through the rotor blades contribute to reduce the conversion efficiency. Finally, in our measurements the centrifugal pump has its own efficiency that affects also the global energy conversion process. All these effects can be included in the average efficiency $\eta$ of the apparatus, which is defined as

$$
\eta=P_{\mathrm{e}} / P_{\mathrm{nom}}
$$

where $P_{\mathrm{e}}$ is the extracted power and $P_{\text {nom }}$ is the impinging power of the wind given by equation (4).

Modern wind turbines start working when the wind velocity is $4-5 \mathrm{~m} \mathrm{~s}^{-1}$, reach the maximum efficiency for a wind velocity of about $10-15 \mathrm{~m} \mathrm{~s}^{-1}$ and stop when the wind velocity reaches values greater than $20-25 \mathrm{~m} \mathrm{~s}^{-1}$; their efficiency ranges from about $\eta \approx 0.2$ to about $\eta \approx 0.3$.

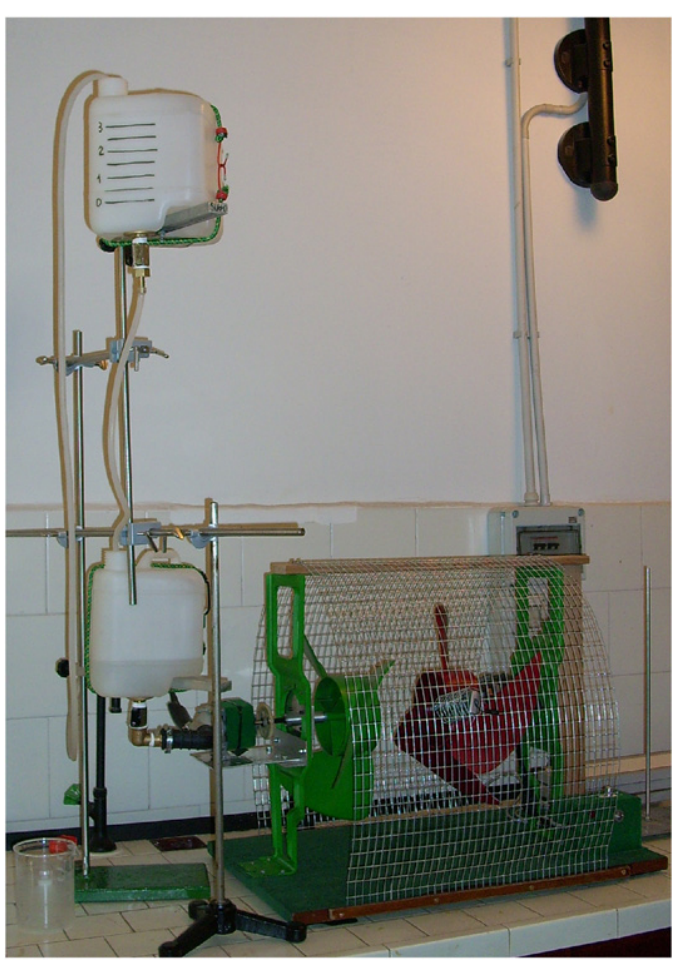

Figure 3. A picture of the experimental apparatus consisting of two fans, a small hydraulic pump and two water tanks.

\section{Experimental apparatus and results}

To perform the experiment, we built a prototype of a windmill by using two fans of $38 \mathrm{~cm}$ in diameter, a small hydraulic centrifugal pump and two five-litre water tanks. The primary fan is used to generate a useful air flow with controlled velocity; it is powered by an electric motor with variable angular velocity. The velocity of the wind is measured by an anemometer with an accuracy of about $5 \%$ of the reading. The secondary fan is used to extract energy from the air flowing through it; it is connected to a small centrifugal hydraulic pump that pumps water from a tank, located at the same level as the pump, to another one located $h=1 \mathrm{~m}$ higher than the previous one. On the lateral surface of the latter tank, a scale is marked that allows one to measure the volume of pumped water as a function of time. Figure 3 shows the experimental apparatus.

The experiment was performed by measuring the quantity of water pumped as a function of time at different wind velocities, which were set 


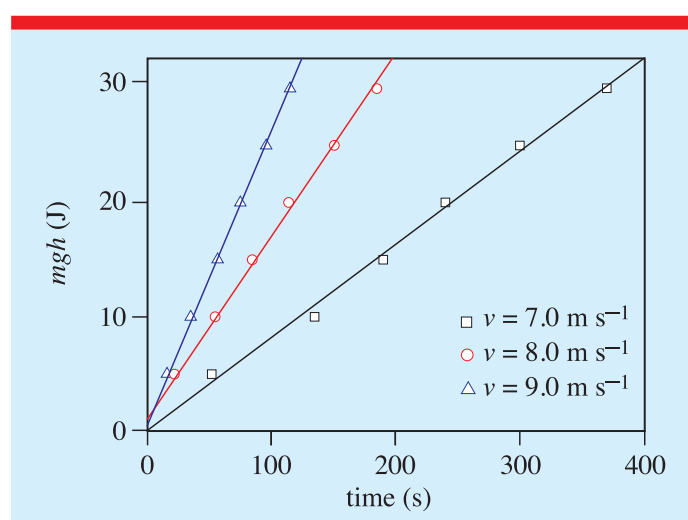

Figure 4. Variation of the potential energy of the pumped water as a function of time for different wind velocities. Symbols are experimental data and lines are best-fit curves.

by changing the angular velocity of the primary fan. Typical results are shown in figure 4 .

\section{Discussion and conclusion}

The measurements performed allowed us to determine the power extracted by the rotor from the wind. Considering that the height to which the water rises is $h=1 \mathrm{~m}$, the gain in potential energy is $\Delta U=\rho_{\mathrm{w}} V g h=m g h\left(\rho_{w}\right.$ is the water density). The slope $\mathrm{d} U / \mathrm{d} t$ indicates the rate at which the potential energy is gained; therefore from the slope of the $\Delta U$ versus time curves we can calculate the extracted power. Multiplying the measured volume $V$ of the pumped water by the fixed factor $\rho_{\mathrm{w}} g h$ one obtains the plots shown in figure 4; the slope of the best-fit curves gives the extracted power. In figure 5, we report the extracted power as a function of the wind velocity.

In 'Theoretical description', we have found that the extracted power from the wind depends on the cubic power of the wind velocity. Therefore, we have fitted the experimental data with the function $f(v)=A+B v^{3}$. The theoretical curve is shown in figure 5. From the fitting one obtains that our apparatus works for velocities greater than $5.2 \mathrm{~m} \mathrm{~s}^{-1}$. This result is mainly due to the presence of mechanical friction.

In order to evaluate the efficiency of the apparatus, we calculate the ratio between the extracted power and the corresponding ideal power of equation (4). The obtained efficiency is about $5 \%$, evaluated at $v=9 \mathrm{~m} \mathrm{~s}^{-1}$. At lower

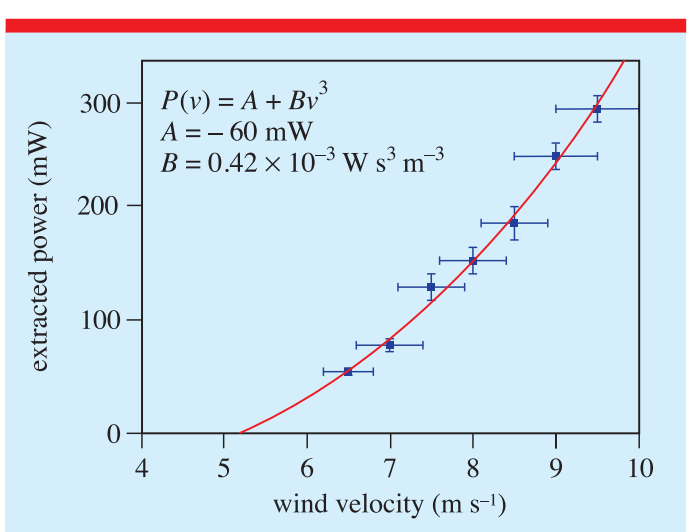

Figure 5. Extracted power as a function of the wind velocity. Symbols are experimental data and the line is a fit obtained as explained in the text.

velocities, the efficiency reduces and eventually becomes zero at $v=5.2 \mathrm{~m} \mathrm{~s}^{-1}$. For a practical wind turbine, losses in efficiency are mainly due to mechanical-power losses in the transmission and pump systems, turbulence and swirl imparted by the rotor to the air flow and viscous drag on the rotor blades.

In conclusion, we have carried out an experiment on wind energy performed with a home-made windmill to pump water from a lower level to a higher one. This activity was carried out with high-school students, aiming at exploiting the wind energy to produce mechanical work by pumping water uphill.

We propose a simple but quantitative experiment, which can be addressed to students beginning their studies on physics, with their knowledge of physics limited to mechanics; this consideration suggested to us the development of suitable apparatus for measurement of the extracted wind energy by using the concept of the mechanical potential energy gained by water and not by involving concepts of electrical energy. From the pedagogical point of view, this feature should not be overlooked; the feasibility of the experiment is rather simple and allows the teacher to discuss renewable energy solely from a mechanical point of view.

Finally, we suggest the inclusion of wind energy topics in school programmes to give students the possibility to gain experience with wind energy. Our aim is to stimulate physics teachers to explain this subject in their classrooms, to 
An experiment on wind energy

improve learning through experimental activities and increase students' awareness of renewable energy sources. Furthermore, we believe that the presentation of the rather abstract concept of energy can benefit greatly from the use of topics like that presented here and may be considered to be of deep social impact by young pupils.

\section{Acknowledgments}

This work was carried out in the framework of the Italian National Plan for Scientific Degrees, under the financial support of the Italian Ministry of Education, University and Research. The authors thank V Mazzola for technical assistance.

Received 25 July 2012, in final form 3 August 2012 doi:10.1088/0031-9120/47/6/755

\section{References}

[1] Russo L 2004 The Forgotten Revolution (Berlin: Springer)

[2] Gipe P 2004 Wind Power: Renewable Energy for Home, Farm and Business (White River Junction, VT: Chelsea Green

[3] Rau M 2011 Moja island: learning about renewable energy sources Sci. Sch. 1950

[4] Fiordilino E and Agliolo Gallitto A $2010 \mathrm{Il}$ laboratorio di fisica nel Progetto Lauree Scientifiche (Rome: Aracne)

[5] Agliolo Gallitto A and Fiordilino E 2010 A didactic experiment and model of a flat-plate solar collector Phys. Educ. 46 312-7

[6] Agliolo Gallitto A and Fiordilino E 2007 Progetto Lauree Scientifiche-Fisica dell'Ateneo di Palermo Università e Scuola XII vol 1 pp 39-42

[7] Agliolo Gallitto A 2009 Quattro Anni di Attività del Progetto Lauree Scientifiche-Fisica nell'Ateneo di Palermo Università e Scuola $X I V$ vol 2 pp 28-33

[8] Agliolo Gallitto A 2009 'School adopts an experiment': the magnetic levitation of superconductors Phys. Educ. 45 511-5

[9] Agliolo Gallitto A, Agnello S and Cannas M 2011 'School adopts an experiment': the photoluminescence in extra-virgin olive oil and in tonic water Phys. Educ. 46 599-603

[10] Danish Wind Industry Association www. windpower.org

[11] Patel M R 1999 Wind and Solar Power Systems (New York: CRC)

[12] Betz A 1926 Wind-Energie und ihre Ausnutzung durch Windmühlen (Göttingen: Vandenhoeck)

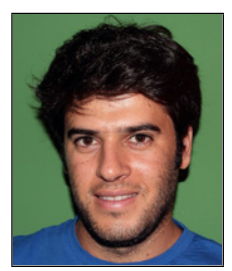

Vincenzo Lombardo is a student of mathematics at the University of Pavia, Italy. He was awarded his mathematics bachelor's degree by the University of Palermo, Italy, defending a thesis that concerned the preparation and realization of the wind energy experiment.

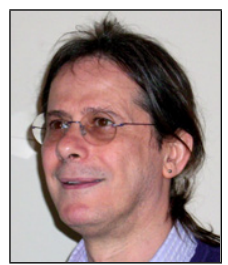

Emilio Fiordilino teaches quantum and statistical mechanics at the University of Palermo, Italy. His research activity mainly concerns the physics of the interaction of strong electromagnetic fields with molecules and nanoparticles, but also volcanism. He spends part of his time lecturing on quantum mechanics in high schools. He is the author of a physics book for secondary schools.

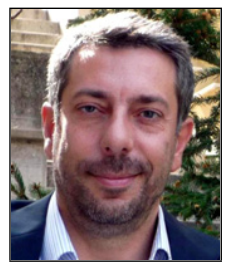

Aurelio Agliolo Gallitto is an associate professor of experimental physics at the University of Palermo, Italy, and works in the field of superconductivity at microwave frequencies. From 2008 to 2010 he was the local coordinator of the Italian National Plan for Scientific Degrees. He is the author of a physics book for secondary schools.

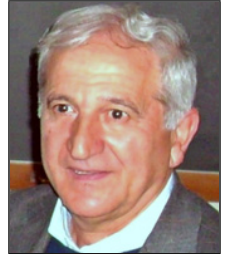

Pasquale Aglieco is a secondary-school teacher of physics. He is involved in the activities of the Italian National Plan for Scientific Degrees-Physics, making a valuable contribution to the organization of activities with schools. 